\title{
Gopher Servers as a Point of Access
}

\author{
by Julie A. Fore ${ }^{1}$ \\ Assistant Automation Librarian \\ Indiana University Ruth Lilly Medical Library
}

This paper will discuss why and how to use gopher servers on the internet to provide access to locally developed data. This includes formatting the data, and establishing the links to the data on the server. The responsibilities involved with providing information on the internet will also be discussed.

\section{Reasons to Mount Data on the Internet}

The internet is a vast source of information and chaos, why would anyone want to add to it?

Some possible reasons for mounting data on the internet include:

-The internet allows remote access to the data. The data and its users are not limited to physical locations. This means that people from other institutions, as well as your own, can get to your data.

-The internet allows 24 hours a day, 7 days a week access to your data (barring the usual network outages or maintenance down time for the data server).

-By providing the data on the internet, it is by definition in electronic format. This allows for further manipulation or massaging of the data. It allows users to take advantage of the computer's abilities, such as searching and sorting.

-The internet allows for quick and easy publishing or updating of your data.

\section{Reasons Not to Mount Data on the Internet}

Most of the reasons for not mounting data on the internet are based on privacy and legal issues.

-The data is copyrighted and can not be re-distributed.

-The data is of sensitive nature and should not be accessible to just anyone, i.e. the world.

-The data is already out on the internet, in a number of different places.

-The internet location for your data is unreliable or not maintained by anyone.

\section{Why Use a Gopher Server}

Currently, gopher clients are widely distributed across the internet community and are available for most types of computer hardware and operating systems. Most gopher client and gopher server software does not require high level computers on which to run, unlike other internet tools such as Mosaic. Gopher clients, as a rule, are easy to use. They provide a common interface to many different types of resources. The Gopher protocol provides the capability to perform searches on databases and files. Currently, this is mostly primitive string or character-by-character searches. Gopher servers have the ability to link or point to other Gopher servers. This linking ability makes it relatively easy to create subject oriented gopher servers. 
Indiana University Ruth Lilly Medical Library Gopher Server Pilot Project

The Indiana University Ruth Lilly Medical Library has been maintaining a database of its Permanent Reserve Collection holdings using a bibliographic database management system called Pro-Cite, made by Personal Bibliographic Systems (PBS). The Library uses Pro-Cite to keep this database because the software allows the Library to provide a number of different printouts for the library patrons to use. The Reserve Collection is shelved (mainly) in title order. Pro-Cite allows the Library to generate printouts of the collection in shelflist (title) order, as well as lists sorted by author or subject heading. (fig. 1) The Library patrons make great use of these printouts, as do the Library Circulation Staff.

\section{RESERVES Collection by AUTHOR}

Abbas, Abul K.

Cellular and molecular immunology.

Abdellah, Faye G.

Patient-centered approaches to nursing.

New directions in patient-centered nursing; guidelines for systems of service, education, and research.

Ackermann, Uwe.

Essentials of human physiology.

$[\ldots]$

Fig. 1 - "Reserve Collection by Author" - Printout from Pro-Cite.

The Permanent Reserve Collection database is small at about 225 records. This made it a perfect pilot for testing how well the Library's various Pro-Cite databases would make the transition from in-house use only to internet accessible information.

The pilot project started with an analysis of the data and data fields already in the Pro-Cite database. (fig 2)

Rec\# 780

Auth Abbas, Abul K.//Lichtman, Andrew H.//Pober, Jordan S.

Titl Cellular and molecular immunology

PIPu Philadelphia

Publ Saunders

Date 1991

Extn xi, $417 \mathrm{p}$

ISBN 0721630324

Call QW 568 A122c 1991

Desc Cellular immunity/Immunity—Molecular aspects/Immunity, Cellular/

Lymphocytes- -immunology

\section{Fig. 2 - Example of a bibliographic record in Pro-Cite}


After the evaluation of the electronic data, a decision was made as to which data elements would be most valuable to a person accessing the database over the internet. I decided to use basic bibliographic citation fields, i.e. author, title, place of publication, publisher and date; as well as the subject heading information. The data from these six fields were then exported from Pro-Cite using Pro-Cite's import/export utilities. Pro-Cite created a standard comma ( ",") delimited file. (fig 3.)

“Abbas, Abul K./ / Lichtman, Andrew H./ / Pober, Jordan S.", "Cellular and molecular immunology","Philadelphia","Saunders","1991","Cellular immunity/Immunity-Molecular aspects / Immunity,Cellular/Lymphocytes-immunology"

"Abdellah, Faye G.","Patient-centered approaches to nursing",,"New

York", "Macmillan", "<1960>","Nurse-patient relations/Education, Nursing"

Fig. 3 - Sample of Pro-Cite Export File in Comma Delimited Format.

The Gopher Server software being used by the Ruth Lilly Medical Library, KA9Q NOS, requires database files to be in dBASE III or dBASE IV format. While most current database management programs such as Paradox by Borland and R:BASE by Microrim can save data in dBASE III format, we chose to use the dBASE III program for the next part of the pilot program.

A database structure was created in dBASE III using the six fields exported from the Pro-Cite database. To keep things simple, the Pro-Cite field labels were used as the field labels in the dBASE database. While Pro-Cite, for the most part, does not use fixed field lengths, dBASE requires fixed field lengths. We made educated guesstimates on what the dBASE field lengths should be. Figure 4 shows the final structure for the Reserves dBASE database.

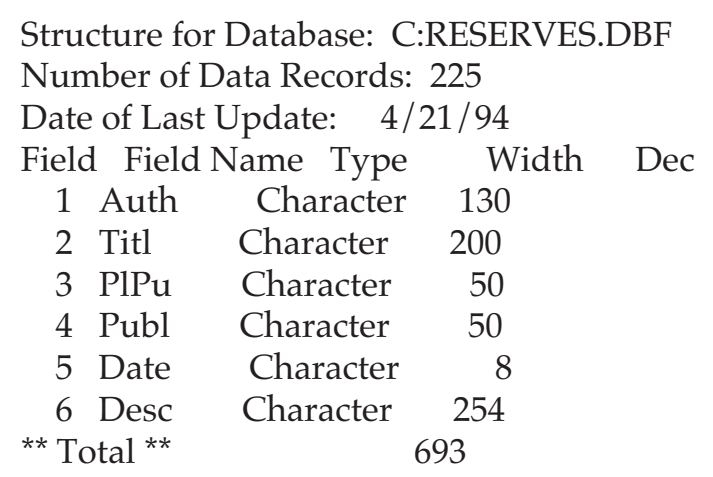

Fig. 4 - Final dBASE III File Structure

The dBASE III import function was used to convert the Pro-Cite produced comma-delimited file into a dBASE III database. A paper report of the new database was then created to verify two things. First, that the data was correctly transmitted from Pro-Cite to dBASE III. Second, to verify that the data, itself, was correct and complete. The data had indeed transferred correctly, but it was found that some of the records in the original database contained incomplete information.

Once the dBASE database had been cleaned up, and an ascii text file bibliography was generated from it using R\&R Report Writer by Concentric Data Systems, the data was ready to be transferred to the actual microcomputer running the gopher server software. In the case of the Ruth Lilly Medical Library Gopher Server, this meant taking down the gopher 
server, that is, exit out of the server program. Then, using the DOS copy command to move the files from the Library's Novell file server to the gopher server's DOS-based microcomputer. Once the actual files were residing on the gopher server's hard drive, a suitable access point in the gopher's menu structure had to be found. Finally, the gopher server's menu configuration files had to be edited to include the pointers to the files.

The most logical place to include the reserve collection information was in the menu with all the other files specific to the Ruth Lilly Medical Library, i.e. the files containing the Library's hours, policies, journal holdings, etc... (fig. 5)

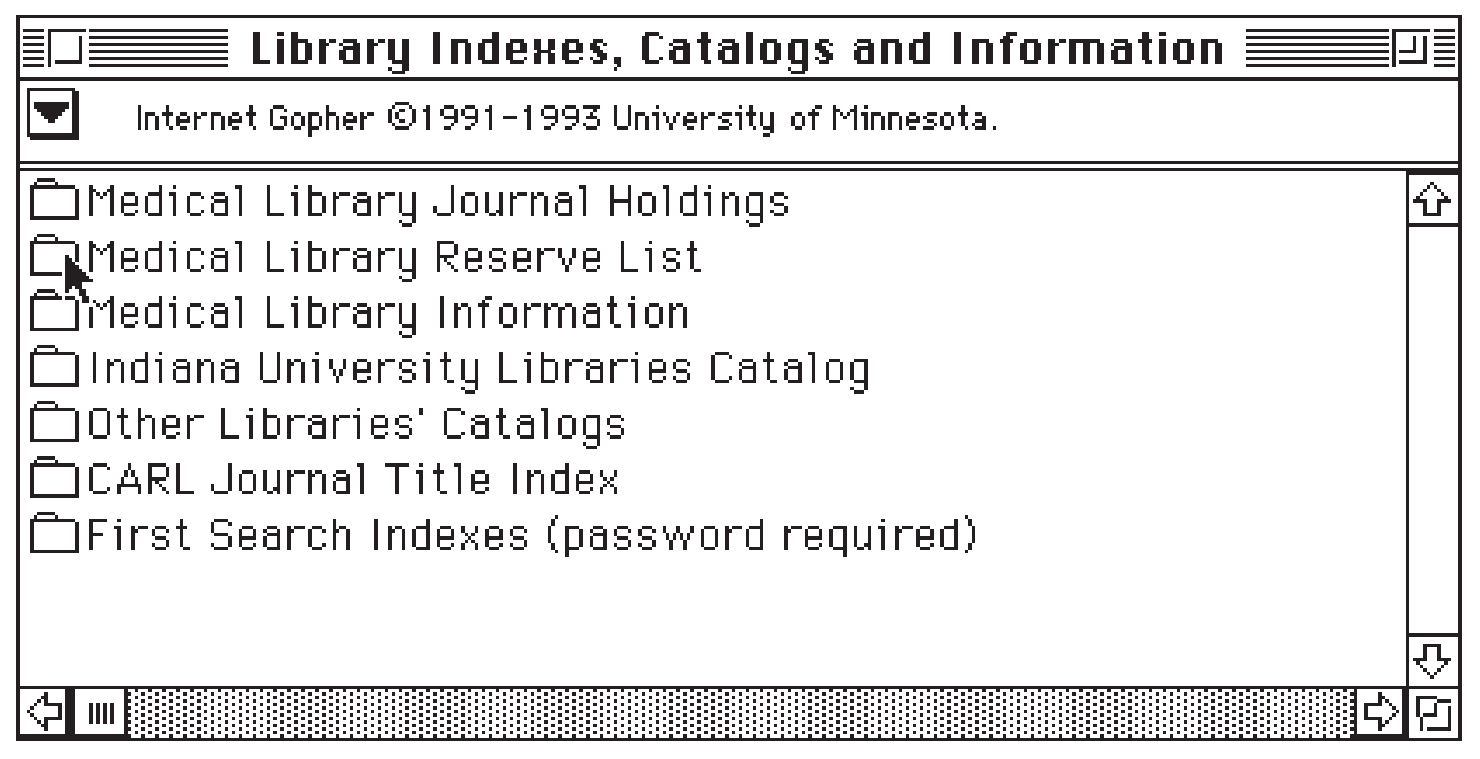

Fig. 5 - "Library Indexes, Catalogs and Information" Menu from Ruth Lilly Medical Library Gopher

In the KA9Q gopher server software, the menus are designed using directories and subdirectories on the server's hard drive and "GINFO" files (which possibly stands for "gopher information" or "gopher index file"). For every menu on the server (seen by a gopher client) there is a corresponding directory on the hard drive of the gopher server and in that directory a GINFO file. The GINFO file contains five elements: 1) the text shown on the menu to a gopher client, 2) a code for the type of resource that is being pointed to (text file, database, directory, Macintosh Binhexed file, uuencoded file, GIF file, etc...), 3) the name and path of that resource (for example /server/reserve.db/reserves.dbf), 4) the internet address of the gopher server that provides the resource (for example gopher.medlib.iupui.edu) and finally, 5) the port for that gopher server, usually port 70. An example of a GINFO file is seen in figure 6. The GINFO file is were the telnet or ftp links to other internet sites are described.

1Medical Library Reserve List

1Medical Library Information

1Medical Library Journal Holdings

1Indiana University Libraries Catalog

1Other Libraries' Catalogs

1CARL Journal Title Index

1First Search Indexes (password required) 1c:/library/FIRST 134.68.85.17 1c:/server/reserve.db gopher.medlib.iupui.edu 70

1c:/server gopher.medlib.iupui.edu 70

1c:/pub gopher.medlib.iupui.edu $\quad 70$

1c:/pop/catalog gopher.medlib.iupui.edu 70

1/Libraries yaleinfo.yale.edu 7000

1c:/library/CARL gopher.medlib.iupui.edu 70

Fig. 6 GINFO File for the "Library Indexes, ...." Menu of the Ruth Lilly Medical Library Gopher Server. 
Figure 7 illustrates what might be found in a directory on a gopher server, note the presence of the GINFO file.

$$
\begin{aligned}
& \text { Volume in drive } \mathrm{M} \text { is BVOL } \\
& \text { Directory of M: \GOPHER \LIBRARY } \\
& \text { GINFO } \quad 523 \text { 05-13-94 8:48p } \\
& \text { CARL } \quad<\text { DIR }>\quad 05-23-94 \text { 10:35a } \\
& \text { FIRST } \quad<\text { DIR }>\quad 05-23-94 \text { 10:35a } \\
& 3 \text { file(s) } 523 \text { bytes } \\
& \text { 191,299,584 bytes free }
\end{aligned}
$$

Fig. 7 - Listing of the files in the Sub-Directory containing the GINFO file from Figure 6.

The second line of the GINFO file shown in figure 6 is the pointer to the Library's Reserve Collection Menu. As one moves through the Gopher's menu structure, the Medical Library Reserve List Menu eventually appears. (fig. 8)

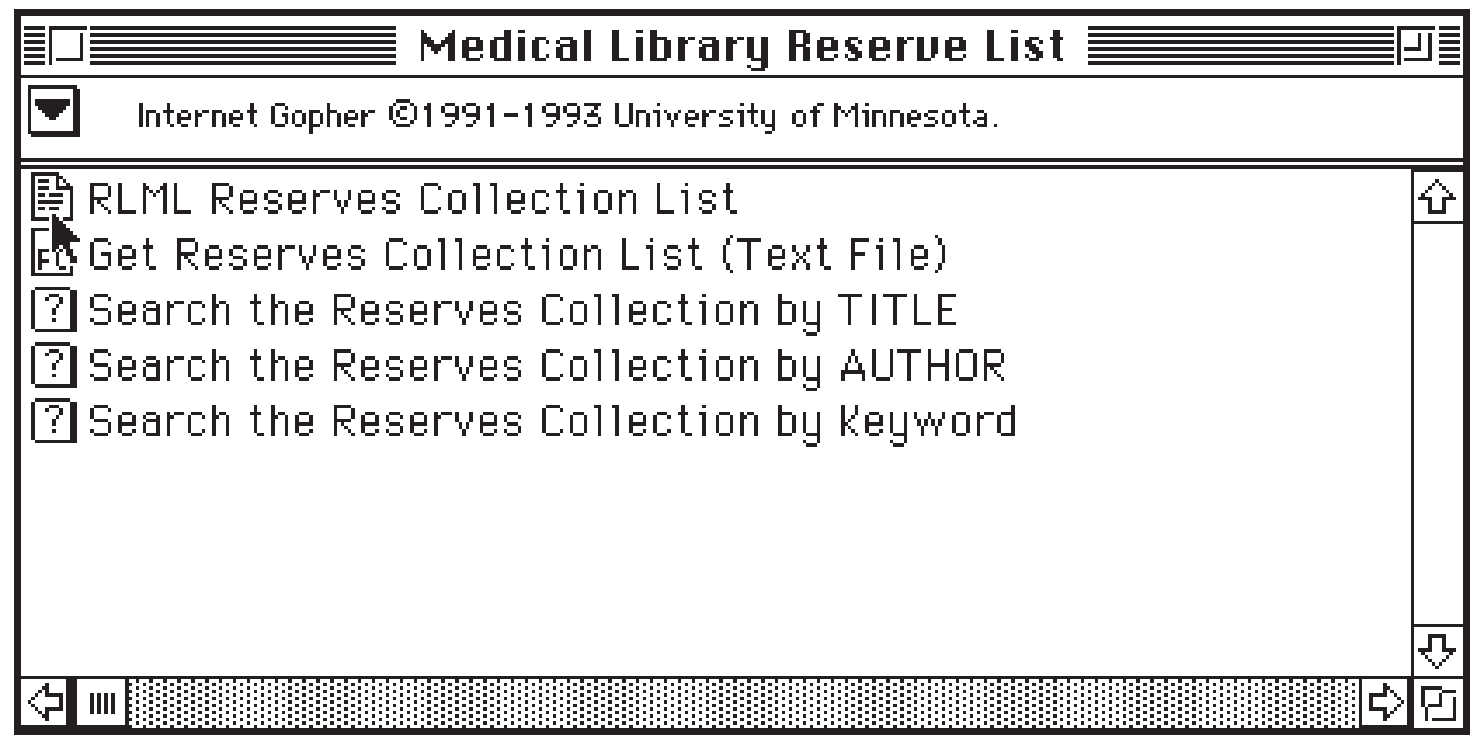

Fig. 8 - "Medical Library Reserve List" Menu of the Ruth Lilly Medical Library Gopher Server.

The "Medical Library Reserve List" menu allows a gopher client to browse or page through a text file bibliography of the Reserve Collection, ftp (file transfer protocol) the bibliography back to the user, or perform a character search on the database either using the title field, the author field, or the descriptor field. The GINFO file for this menu determines which function is performed on which file. There are only three files in the directory for this menu. The GINFO file, the actual database file called reserves.dbf, and the text file bibliography called reserves.txt. (fig. 9) 
Volume in drive $\mathrm{M}$ is BVOL

Directory of M:\GOPHER \SERVER \RESERVE.DB

\begin{tabular}{lcc} 
RESERVES DBF & $156,16004-21-94$ & $3: 46 \mathrm{p}$ \\
RESERVES TXT & $30,98904-25-94$ & $4: 17 \mathrm{p}$ \\
GINFO & $53005-21-94$ & $2: 12 \mathrm{p}$ \\
4 file(s) & 188,210 bytes \\
\multicolumn{1}{c}{$191,299,584$ bytes free }
\end{tabular}

Fig. 9 - Directory listing of the RESERVE.DB subdirectory

Figure 10 illustrates the GINFO file for the Medical Library Reserve List menu.

ORLML Reserves Collection List 0c:/ server/reserve.db/reserves.txt 70

5Get Reserves Collection List (Text File)

gopher.medlib.iupui.edu $\quad 70$

7Search the Reserves Collection by TITLE

gopher.medlib.iupui.edu 70

5C:/SERVER/ reserve.db/reserves.txt

7Search the Reserves Collection by AUTHOR qc:/server/reserve.db/reserves.dbf AUTH

gopher.medlib.iupui.edu 70

7Search the Reserves Collection by Keyword qc: / server/reserve.db/reserves.dbf DESC

gopher.medlib.iupui.edu

70

Fig. 10 - GINFO for "Medical Library Reserve List" menu

Figure 11 shows what the text file bibliography looks like when viewed by a gopher client. The bibliography file was created so that the users could have access to a formatted file that they could browse through. It was decided that the dBASE database format was not very easy to browse (fig. 13), nor was it in a file format that most people could use once they had it back at their own computer. The double slash marks (//) in the author field are left over formatting codes from Pro-Cite. These codes will be removed the next time the database needs significant updating. 


\begin{tabular}{|c|}
\hline 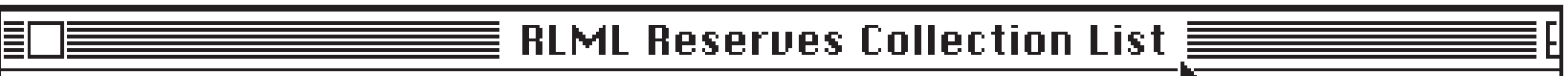 \\
\hline $\begin{array}{l}\text { Abbas, Abul K//Lichtman, Andrew H.//Pober, Jordan S. } \\
\text { Cellular and molecular immunology. } \\
\text { Philadelphia: Sanders, } 1991 .\end{array}$ \\
\hline $\begin{array}{l}\text { Abdellah, Faye G. } \\
\text { Patient-centered approaches to nursing. } \\
\text { New Vork: Macmilian, } 1960 \text {. }\end{array}$ \\
\hline $\begin{array}{l}\text { Abdellah, Faye G.//Bailey, June } T \text {. } \\
\text { New directions in patient-centered nursing; guidelines for systems of } \\
\text { service, education, and research. } \\
\text { New York: Macmilan, } 1973 \text {. }\end{array}$ \\
\hline
\end{tabular}

Fig. 11 - Browsing the "RLML Reserves Collection List" Option

The KA9Q gopher server search capabilities are currently string or character based searches. This means that when "Search the Reserve Collection by AUTHOR" is selected off the "Medical Library Reserve List" menu, a dialog box will appear asking the user to enter the words to be searched for in the author field of the database. In the example illustrated by figures 12 and 13, the user asked the gopher to search for all occurrences of the word "sid" in the author field. As the results of the search show (fig. 13), the gopher does not care where the "word" "sid" appears in the author field. It found the letters, or characters, "s-i-d" in the word "President" and in "Sidney". It is expected that gopher-based searching will improve in the future. If not, then some other internet tool will take gopher's place.

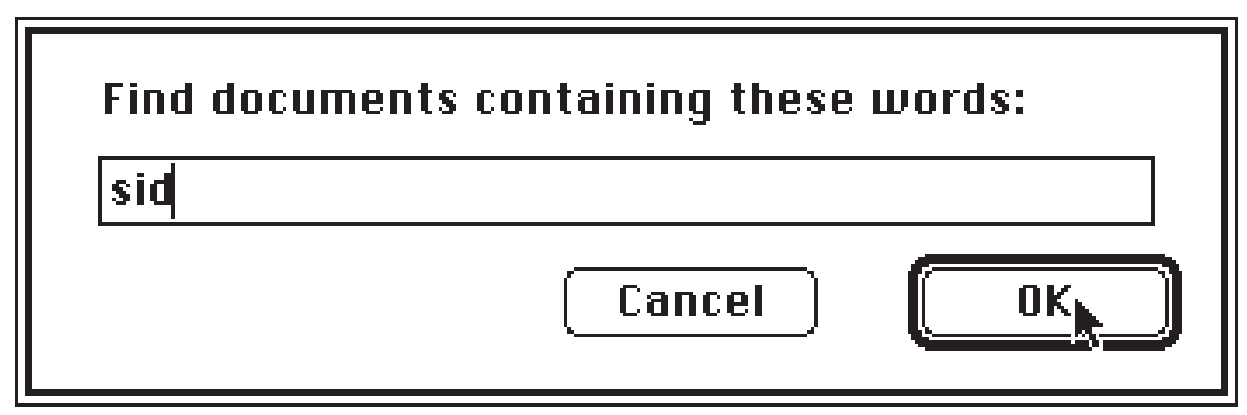

Fig. 12 - Gopher Search Dialog Box 


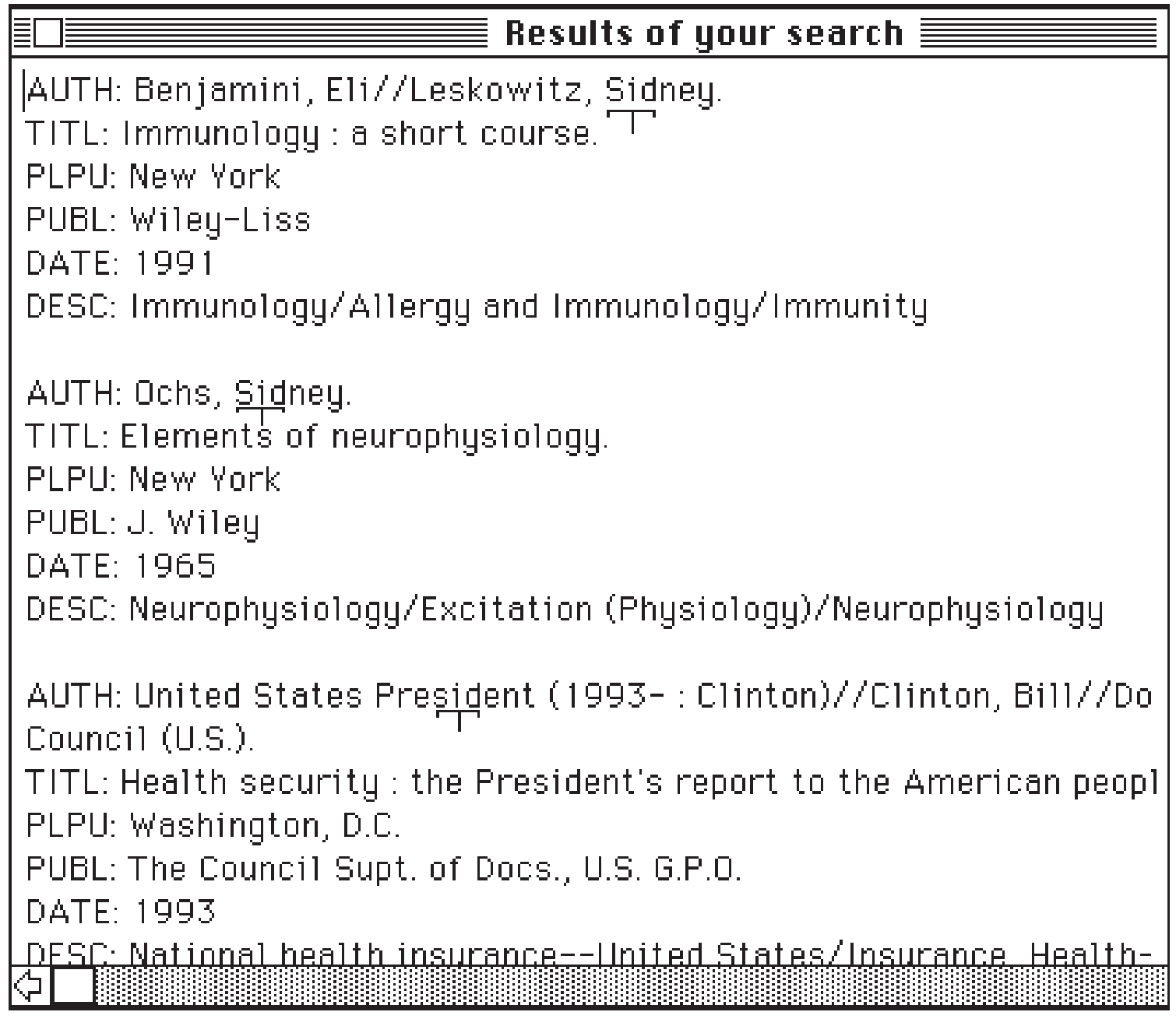

Fig. 13 - Results of Searching for "Sid" in the Author Field of the Reserve Collection Database

\section{Internet Responsibility}

It is not enough to just mount a database on the internet. It is necesary to take responsibility for it and for the gopher server on which it resides. There are a number of points to keep in mind when setting up and maintaining servers on the internet.

-Keep your server up and running. No one can use your data if your server or your network is down.

-When (not if) you take your server down for routine maintenance, i.e. on a routine schedule, post this information on your server. 
-If there are limitations to your server or your data, post this on your server and on any public announcements you send out about your server. Some examples of limitations might include, access only during non-business hours like 5 p.m - 6 a.m EST, a limited number of simultaneous users, the fact that passwords are required for access to certain files or services, or that only users from a certain place (campus, university, etc...) are permitted access to a resource.

-Keep your data current and accurate. If this is not possible, indicate on the server that the data is old/out-of-date or not necessarily accurate.

-If you move your resource to a new internet site or remove it from the internet, announce this. Place a notice stating the new location of the resource in the old location of the resource. Post announcements to appropriate LISTSERVs and newsgroups.

-If you are keeping copies (mirrors) of your resource at more that one location, keep them current and announce their locations as well.

Technical Information about the Indiana University Ruth Lilly Medical Library Gopher Server URL: gopher://gopher.medlib.iupui.edu port 70

The IU RLML Gopher Server is currently running on a Gateway 2000 386-25 MHz processor with 4 MB of RAM. The computer has a $300 \mathrm{MB}$ Hard Disk, of which approximately $50 \mathrm{MB}$ is being used. The computer is running DOS Version 5.0 and is attached to a 4 Mbps Token Ring LAN. The Server is backed up weekly to a Novell Netware 3.11 file server.

The Gopher Server Operating System is KA9Q NOS, a DOS-based Network Operating System. KA9Q supports Gopher; POP2, POP3, and SMTP mail server protocols; ftp, anonymous ftp, telnet and finger; CSO Name Server functions; NTP (time) Server functions; and WWW Server functions. The Indiana University Ruth Lilly Medical Library currently is not supporting the mail server functions but is experimenting with the other capabilities of the KA9Q software.

In the future, the Indiana University Ruth Lilly Medical Library Gopher Server will be switched to a 10 Mbps Ethernet LAN. It MAY be switched to a UNIX - based computer, and it may be given additional WorldWideWeb (WWW or W3) functionality and resources.

\section{Places to find more information}

Newsgroups for gophers and other information servers:

comp.infosystems.gopher

comp.infosystems.www

comp.infosystems.wais

Frequently Asked Question (FAQ):

Gopher FAQ can be retrieved via anonymous ftp from the following site:

rtfm.mit.edu:/pub/usenet/news.answers/gopher-faq

or via gopher from:

129.130.10.5 port=70, path=0/Frequently Asked Questions (FAQ)/gopher-faq

KA9Q NOS (Network Operating System) DOS-based Gopher Server Software.

KA9Q Mailing List:

send an email to Ashok 
ashok@biochemistry.cwru.edu and ask to be added to the mailing list. This address is an individual, so be nice.

KA9Q Manual:

The User Manual is available via gopher from the following site:

cases.pubaf.washington.edu, port 70, in 1c:Imanual

University of Minnesota — The Top Gopher:

gopher://gopher.tc.umn.edu port 70

Questions or Comments for the Gopher development team, send e-mail to:

gopher@boombox.micro.umn.edu

News about new gopher servers and software, subcribe to the gopher-news mailing list:

gopher-news-request@boombox.micro.umn.edu

The most recent releases of gopher software is available via anonymous ftp from:

boombox.micro.umn.edu in the /pub/gopher directory.

1. Paper presented at IASSIST 94 in San Francisco, May 1994 